\title{
Routing and Mobility Management in the Internet of Things
}

\author{
Bruno P. Santos ${ }^{12}$, Luiz Filipe M. Vieira ${ }^{2}$, Antonio A. F. Loureiro ${ }^{2}$ \\ ${ }^{1}$ Departamento de Computação e Sistemas (DECSI) \\ Universidade Federal de Ouro Preto (UFOP) \\ João Monlevade - MG - Brasil \\ ${ }^{2}$ Departamento de Ciência da Computação (DCC) \\ Universidade Federal de Minas Gerais \\ Belo Horizonte - MG - Brasil \\ bruno.ps@ufop.edu.br, \{bruno.ps, lfvieira, loureiro\}@dcc.ufmg.br
}

\begin{abstract}
This Ph.D. Thesis proposes new techniques for routing and mobility management for Internet of Things (IoT). In the future IoT, everyday mobile objects will probably be connected to the Internet. Currently, static IoT's devices have already been connected, but handle mobile devices suitably still being an open issue in IoT context. Then, solutions for routing mobility detection, handover, and mobility management are proposed through an algorithm that integrates Machine Learning (ML) and mobility metrics to figure out devices' mobility events, which we named Dribble. Also, an IPv6 hierarchical routing protocol named Mobile Matrix to boost efficient (memory and fault tolerance) end-to-end connectivity over mobility scenarios. The Thesis contributions are supported by numerous peer-reviewed publications in national and international conferences and journals included in ISI-JCR. Also, the applicability of this Thesis is evident by showing that our results overcome state-of-the-art in static and mobile scenarios, as well as, the impact of the proposed solutions is a step forward in at least two new research areas so-called Internet of Mobile Things (IoMT) and Social Internet of Things (SIoT), where devices move around and do social ties respectively. Moreover, during the Ph.D. degree, the author has contributed to different computer network fields rather than routing by publishing in areas like social networks, smart cities, intelligent transportation systems, softwaredefined networks, and parallel computing.
\end{abstract}

\section{Nature of the Problem and its Relevance}

The explosive growing number of everyday objects connected to the Internet emerges as a result of rapid progress and adoption of embedded systems, wired and wireless communication, and mainly the use of devices able to sense and move in the environment. The set of objects or just "things" placed within the cyber-physical environment, where they can sense, interact with one each other and with humans is the Internet of Things (IoT) [Vasseur and Dunkels 2010]. Mobility is a major factor present in our daily life and, thus, "things" also should be able to move around forming the so-called Internet of Mobile Things (IoMT) [Nahrstedt et al. 2016]. In the future, the Social Internet of Things (SIoT) will be reality where the smart devices do social ties among themselves as well as with humans in the social cyber-physical environment [Afzal et al. 2019]. Those new technological artifacts have the potential to change the Internet as we see nowadays 
and how entities (machines and humans) interact one each other towards ubiquitous environments, where huge research and commercial opportunities emerges. The mobility is present in everyday life making our life easier and turns IoT applications more flexible. By extending IoT to handle mobility, IoT becomes even more ubiquitous.

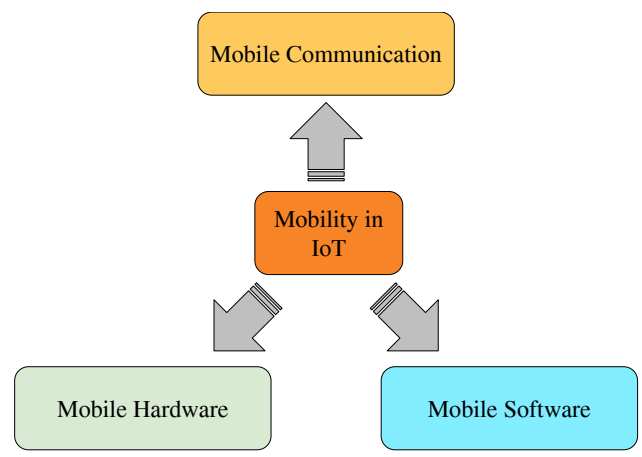

(a) Requirements for a Mobile loT.



(b) Building block operations to handle mobility.

Figure 1. Thesis context.

Many solutions have been proposed to support static IoT. Standards were already done in the same context. However, handle mobility suitably remains an open issue in IoT [Narendra and Misra 2016]. By supporting mobility, it is expected that the "things" to be transported or move themselves during normal usage without inhibit its normal operation and communication exchanges. Figure 1a shows the requirements to achieve a mobile IoT. Mobile Communication usually encompass ad hoc networks as well as infrastructured networks. Thus, every network stack layer plays an essential role in mobile communication. Mobile Hardware comprises of mobile devices able to move by themselves or being attached to a mobile entity. The mobile pattern of the devices and humans can profoundly influence in mobile communication. Thus, this understatement is also an issue [Asl et al. 2013]. Mobile Software deals with the characteristics and requirements of mobile applications. For example, an application may require specific types of communication patterns to provide its practical value to humans. These characteristics and requirements usually pose challenges to the mobile IoT solution designs.

New IoT mobile-based paradigms already have notoriety in a wide range of domains (e.g., smart home, vehicles, and cities) [Al-Fuqaha et al. 2015]. Several authors have surveyed a bulk of applications and challenges in such IoMT context showing the relevance and applicability of this study [Agnihotri and Ramkumar 2017, Nahrstedt et al. 2016].

\section{Goals}

Our focus is to propose advances in mobility-aware solutions for IoT, especially mobile communication solutions. In this context, the performance improvement and real adoption of mobile IoT's applications are heavily influenced by the full mobility awareness of the network stack. From the lens of the network stack, we argue that the routing layer plays a fundamental role in the proper functioning, scalability, and identification of objects, especially regarding the highly dynamic network of "things".

For these reasons, the Thesis' scope is the routing layer, but we were not limited to it. Indeed, we have investigated three basic operations to handle mobility in IoT: Mobility 
Detection, Handover, and Mobility Management processes. Figure $1 \mathrm{~b}$ highlights those operations and their relationship. Such operations can be explored through different layers in the network stack and can be influenced by endogenous and exogenous factors, such as the device mobility patterns and human intervention, respectively. Mobility Detection is the task of figuring out when a mobile entity is moving, specifically in our study within the network. The handover occurs when the entity changes its actual attachment point to another one. Mobility Management is the task of keeping track of the current location of a mobile entity. These basic operations raise many specific challenges in IoT, for which solutions must be proposed: (i) One specific challenge in Mobility Detection is that frequently IoT devices have only basic components (e.g., processing and communication units), thus how can the network stack protocols figure out that the entity is moving? Note that protocols may not have dedicated location hardware to query this information. (ii) regarding mobile handover and management, from the lens of routing, how to promote transparent mobility hiding changes from upper layers (e.g., address changes)?

\section{Relevant Theory and Approach}

In Chapters 2 and 3, we begin by reviewing related work. First, we concentrate on the basics by giving an IoT's overview through its historical perspective and why to study this subject. Also, we highlight the basic building blocks and the mobility in the IoT context. Then, the experimental framework to be used in this Thesis is described in detail. We have employed the widely accepted IPv6 over Low-Power Wireless Personal Area Networks (6LoWPAN) to address devices, then exploited the hierarchical addressing nature to boosts memory and fault tolerance efficiency in the routing management over mobile scenarios. As contributions in the building blocks presented in Figure 1b (i) a machine learn-based timer schemer selector was proposed, which we named as Dribble ${ }^{1}$. Mostly of routing protocols for mobile IoT have one timer scheme that governs the communication structure construction and maintenance by triggering from time to time control advertisements. The timers must deal fairly with a basic trade-off as show in Figure 2a: if it is too greedy by sending advertisement frequently, it responds quickly to topology changes, but it spends energy and introduces an overhead to the wireless shared channel. Dribble handles this by learning the device's mobility pattern and then it selects a proper time scheme in order to have a better the trade-off balance (Desirable case in Figure 2a).

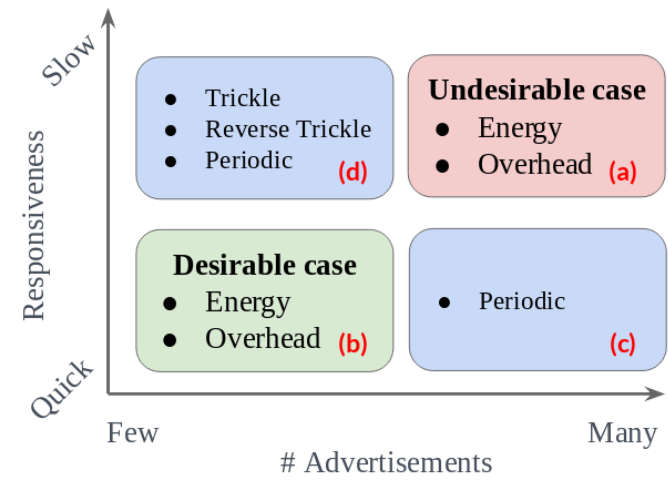

(a) Timer schemes' basic trade-off faced.

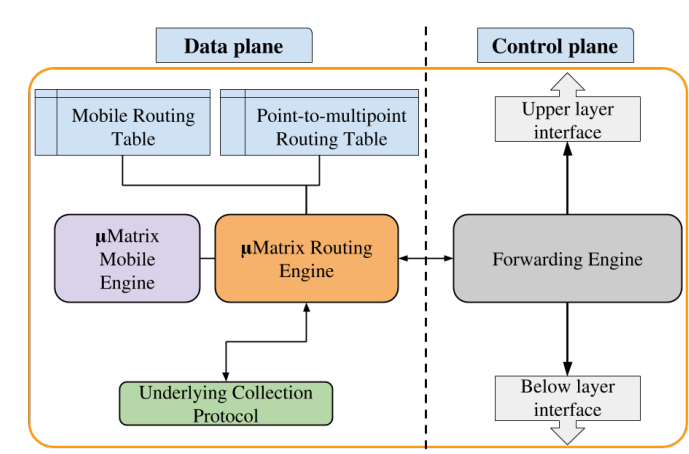

(b) Mobile Matrix protocol's architecture.

Figure 2. Theses approaches.

\footnotetext{
${ }^{1}$ As a reference to Trickle, a well-known timer scheme for figure out network topology changes
} 
The Dissertation then focuses on the description of Matrix and Mobile Matrix routing protocols algorithms in Chapters 5 and 6 respectively. They are contributions in Figure 1b (ii) handover process, and (iii) mobility management. The former, Matrix, it is a novel routing protocol able to provide any-to-any routing under static and temporary faulty topologies. The latter, Mobile Matrix, it inherits all Matrix's features, but it also can run under mobile scenarios. For both, we present their detailed architectures, which is summarized in the Figure 2b. Both architectures have data and control planes and an engine to constructing the routing tables based on a hierarchical addressing scheme. Mobile Matrix distinguishes by adding a Mobile Engine that relies on a Finite State Machine to control the device mobility state. For both algorithms, we derive their complexities costs for routing construction and maintenance through formal algorithm analysis. Also, we have evaluated our approaches against state-of-the-art protocols such as XCTP [Santos et al. 2015], RPL [Winter et al. 2012] and its optimizations [Oliveira and Vazão 2016]. We have evaluated them by simulation over different static and mobile (i.e., human and non-human mobility patterns) scenarios. The results show that Mobile Matrix has the potential to provide support to the next generation SIoT and IoMT paradigms.

Finally, the dissertation is concluded (Chapter 7) by summing up the main results as well as pointing out future directions and open problems. For the latter, we highlight that IoT and 5G will play a key role in the concept of the Internet soon. Also, we argue that we are not prepared yet to support the Social Internet of Things (SIoT) paradigm fully, thus it is an open paradigm to be exploited by researchers and industry.

Table 1. Ph.D. in numbers.

\begin{tabular}{|c|c|c|c|c|c|c|}
\hline Where / Qualis & A1 & A2 & B1 & B2 & Not Found & $\begin{array}{c}\text { Totals } \\
\text { (Thesis/Others) }\end{array}$ \\
\hline Conferences & & 1 & 3 & 2 & & $(3 / 3)$ \\
\hline Journals & 4 & 1 & & & & $(2 / 3)$ \\
\hline Book Chapters & & & & 2 & 1 & $(1 / 2)$ \\
\hline Totals & & & & & & 14 \\
\hline
\end{tabular}

\section{Contributions of the Thesis with Indicative References}

The Thesis has generated a important amount of research contributions, this is evidenced by the number of articles in conferences and journals with ISI-JCR impact factor published (Table 1). Following, we highlight scientific contributions directly related to the Thesis (some publications are repeated in different items the list): (i) Literature review: in order to gather as much information as possible we have surveyed the IoT field. As result we have made a book chapter [Santos et al. 2016] which was the first step of this Ph.D. process. (ii) Novel Methods: we have proposed mainly three novel approaches to handle mobility in IoT context (see Figure 1b). First, Dribble to better figure out mobility event [Santos et al. 2018c], Second, Matrix to hierarchically routing messages for static or temporary faulty IoT networks [Peres et al. 2016, Peres et al. 2018]. Third, Mobile Matrix to manage in-network mobility devices [Santos et al. 2017a, Santos et al. 2018a]. (iii) New experimental studies: there is a lack of available real mobility traces in IoT domain. Thus, we proposed a mix of human and non-human mobility patterns to simulate movements and then evaluate our propositions [Santos et al. 2017a, Santos et al. 2018a]. (iv) Ready to 


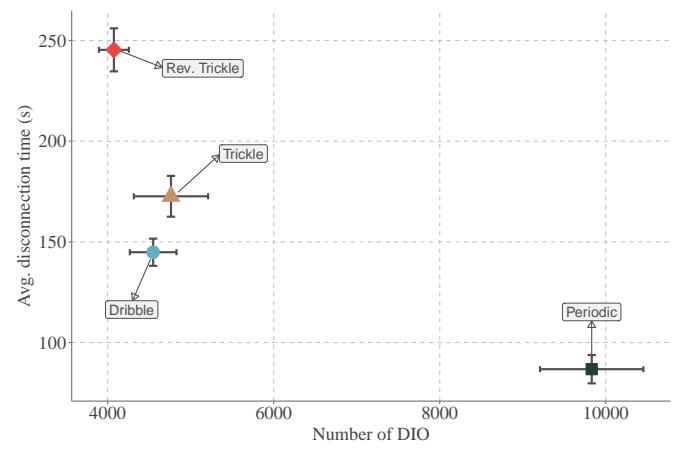

(a) The trade-off between control overhead and disconnection time.

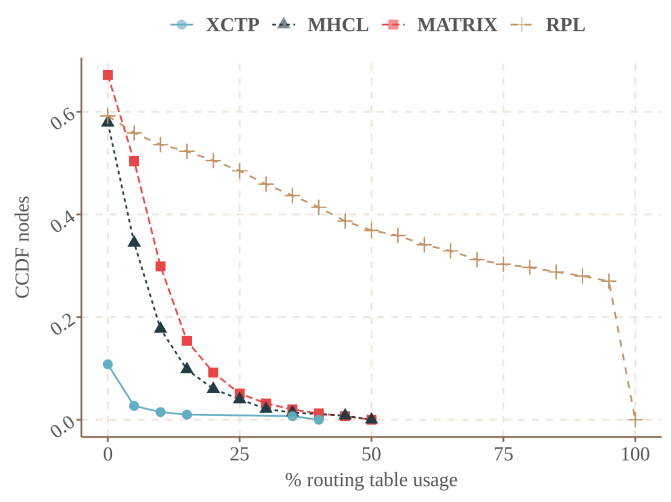

(b) Routing table usage Complementary Cumulative Distribution Function (CCDF). The routing table size was setted to 20 entries.

Figure 3. Theses initial results.

go algorithm: we implement our solutions (Matrix and Mobile Matrix) on Contiki-OS, an Operation System for IoT. This means that our algorithm is read to go to real devices [Peres et al. 2016, Peres et al. 2018, Santos et al. 2017a, Santos et al. 2018a].

Others scientific publication beyond this Thesis scope were in Intelligent Transportation Systems, Social Networks, and IoT/WSN: [Rettore et al. 2016, Cunha et al. 2017a, Cunha et al. 2017b, Santos et al. 2017c, Santos et al. 2018b, Santos et al. 2017b, Rettore et al. 2020]

\section{Results and Analysis}

This section presents the main results of the Thesis. As mentioned, this Thesis aims to improve performance and support of mobility in solutions and applications for IoT.

Dribble is a timer scheme selector. It means that Dribble selects from time to time a timer schemes (e.g., Trickle Timer, Periodic, and others) helping routing protocols to figure out devices' motion events and react upon that. Each scheme is suitable for a mobility pattern, thus Dribble leans the devices' mobility behavior and then updates the schemer properly and timely. The goal is to balance the trade-off faced by timer schemes (Figure 2a), which, in our experiments, Dribble have shown good results. Figure 3a shows traditional schemes and Dribble comparing disconnection timer (response over topology changes) and number of control packets ${ }^{2}$ while others protocols in at least one feature.

We also propose Matrix, a routing protocols for IoT. We build it upon the idea of using hierarchical IPv6 address allocation that explores cycle-free network structures. To sum up, Matrix achieves the following essential goals for routing: (i) Any-to-any routing which enables end-to-end connectivity; (ii) memory efficiency in which Matrix compacts routing tables being scalable to extensive networks; (iii) Reliability of $99 \%$ of delivery rate was achieved without end-to-end mechanisms, and $\geq 90 \%$ of end-to-end when a route exists under challenging networks conditions (faulty tolerance). (iv) Hardware independence and IoT integration, Matrix does not rely on specific radio chip, and Matrix allocates global (and structured) IPV6 addresses to all nodes (compatible with 6LoWPAN) contributing to the realization of the IoT. Figure $3 \mathrm{~b}$ shows routing table usage for

\footnotetext{
${ }^{2}$ We use Routing Protocol for Low-Power and Lossy Networks (RPL) that is the state-of-the-art routing protocol for IoT
} 


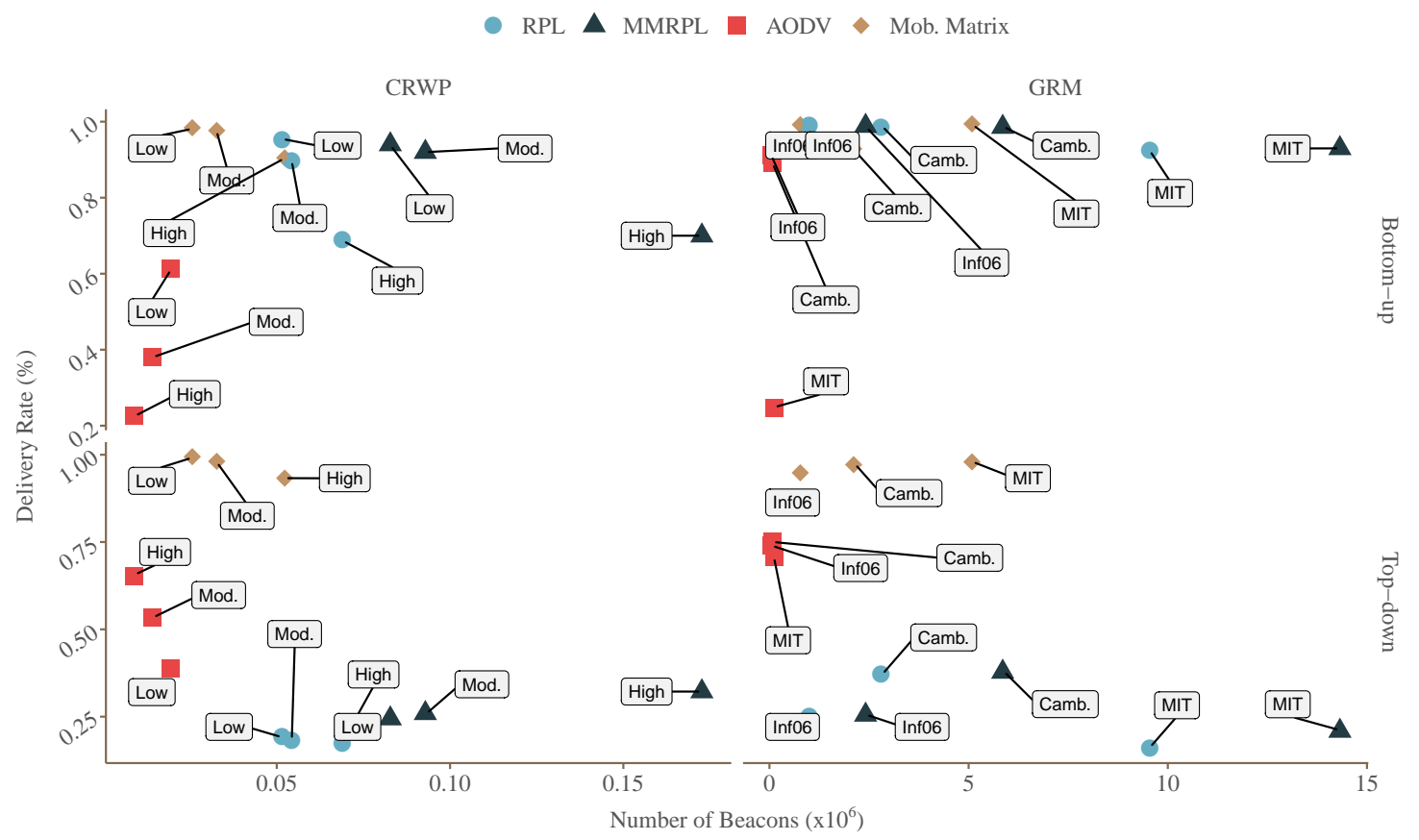

Figure 4. The trade-off between control message overhead and the successful delivery rate.

different routing protocols. RPL is the only protocol that uses $100 \%$ of table entries for some nodes, which quickly fills the available memory and forces packets to be dropped. While XCTP and MHCL show less memory usage, but those protocols do not support any-to-any routing, hardware independence and IoT integration as Matrix. Matrix was simulated in the faulty scenario (Chapter 5). Note that $>35 \%$ of nodes use zero routing table entries meaning that Matrix is more scalable while afford more desirable features.

Mobile Matrix is built upon a previously proposed protocol. Mobile Matrix presents the following features: (i) Transparent mobility management: devices can move in the cyber-physical space without ever changing their IPv6 address; (ii) Optimal routing path distortion: messages addressed to a mobile device, from anywhere in the network, are sent along the shortest path from the source to its current location, using its original IPv6 address; (iii) Passive mobility detection: Mobile Matrix uses Reverse Trickle Timer (RevTT) to detect neighbor device mobility, which can be tuned according to the application or the mobility pattern; (iv) Low memory footprint: Mobile Matrix is in consonance with IPv6 addressing and uses a hierarchical address allocation to reduce memory usage to store routing information in a dynamic mobile environment; (v) No fixed devices required: Mobile Matrix does not rely on fixed devices to manage mobility, except for the border router, commonly employed in 6LoWPAN. Figure 4 shows the performance concerning delivery rate and overhead for Mobile Matrix, RPL and its mobile optimization as well as AODV routing protocols under mobile scenarios ${ }^{3}$. In that plot, high delivery rate and low overhead is desirable. In most situation, Mobile Matrix presents better balance being reliable and overhead efficient.

\footnotetext{
${ }^{3}$ Human and non-human mobility patterns see Thesis Chapter 6
} 


\section{Final Remarks}

We have studied distinct aspects related to routing under mobility in IoT. We did studies, analyses, and formulated proposition for Mobility detection, Handover process, and Mobility Management. Such studies were summed up along this abstract showing that our approaches are alternatives to the status quo of routing in IoT context.

Finally, we would like to thank FAPEMIG, CNPq, and CAPES for their financial support for this research.

\section{References}

Afzal, B., Umair, M., Shah, G. A., and Ahmed, E. (2019). Enabling IoT platforms for social IoT applications: Vision, feature mapping, and challenges. Future Generation Computer Systems, 92:718-731.

Agnihotri, S. and Ramkumar, K. (2017). A survey and comparative analysis of the various routing protocols of Internet of things. International Journal of Pervasive Computing and Communications, 13(3):264-281.

Al-Fuqaha, A., Guizani, M., Mohammadi, M., Aledhari, M., and Ayyash, M. (2015). Internet of things: A survey on enabling technologies, protocols, and applications. IEEE Communications Surveys \& Tutorials, 17(4):2347-2376.

Asl, H. Z., Iera, A., Atzori, L., and Morabito, G. (2013). How often social objects meet each other? Analysis of the properties of a social network of IoT devices based on real data. In Global Communications Conference (GLOBECOM), 2013 IEEE, pages 2804-2809. IEEE.

Cunha, F., Maia, G., Ramos, H., Santos, B. P., Celes, C., Rettore, P., Campolina, A., Guidoni, D., Souza, F., Villas, L., Mini, R., and Loureiro., A. (2017a). Emerging Trends in Vehicular Ad-hoc Network (VANET). Emerging Wireless Communication and Network Technologies: Principle, Paradigm and Performance. Springer Chapter Book.

Cunha, F., Maia, G., Ramos, H., Santos, B. P., Celes, C., Rettore, P., Campolina, A., Guidoni, D., Souza, F., Villas, L., Mini, R., and Loureiro., A. (2017b). Sistemas de Transporte Inteligentes: Conceitos, Aplicações e Desafios (Intelligent Transportation Systems: Concepts, Applications and Challenges - In portuguese). XXXV Simpósio Brasileiro de Redes de Computadores e Sistemas Distribuídos. 1ed.Porto Alegre: Sociedade Brasileira de Computação (SBC) SBRC 2017. Chapter Book.

Nahrstedt, K., Li, H., Nguyen, P., Chang, S., and Vu, L. (2016). Internet of mobile things: Mobility-driven challenges, designs and implementations. In Internet-of-Things Design and Implementation (IoTDI), 2016 IEEE First International Conference on, pages 25-36. IEEE.

Narendra, N. and Misra, P. (2016). Research challenges in the internet of mobile things.

Oliveira, A. and Vazão, T. (2016). Low-power and lossy networks under mobility: A survey. Computer Networks, 107:339-352.

Peres, B., Santos, B. P., de O. Souza, O. A., Goussevskaia, O., Vieira, M. A. M., Vieira, L. F. M., and Loureiro, A. A. F. (2018). Matrix: Multihop Address allocation and dynamic any-To-any Routing for 6LoWPAN. Computer Networks, 140:28 - 40. 
Peres, B. S., Souza, O. A. d. O., Santos, B. P., Junior, E. R. A., Goussevskaia, O., Vieira, M. A. M., Vieira, L. F. M., and Loureiro, A. A. F. (2016). Matrix: Multihop Address Allocation and Dynamic Any-to-Any Routing for 6LoWPAN. In Proceedings of the 19th ACM International Conference on Modeling, Analysis and Simulation of Wireless and Mobile Systems, MSWiM '16, pages 302-309, New York, NY, USA. ACM.

Rettore, P. H., Santos, B. P., Campolina, A. B., Villas, L. A., and Loureiro, A. A. (2016). Towards intra-vehicular sensor data fusion. In Intelligent Transportation Systems (ITSC), 2016 IEEE 19th International Conference on, pages 126-131. IEEE.

Rettore, P. H. L., Santos, B. P., Rigolin F. Lopes, R., Maia, G., Villas, L. A., and Loureiro, A. A. F. (2020). Road data enrichment framework based on heterogeneous data fusion for its. IEEE Transactions on Intelligent Transportation Systems, 21(4):1751-1766.

Santos, B. P., Goussevskaia, O., Vieira, L. F., Vieira, M. A., and Loureiro, A. A. (2018a). Mobile Matrix: Routing under Mobility in IoT, IoMT, and Social IoT. Ad Hoc Networks.

Santos, B. P., Goussevskaia, O., Vieira, L. F. M., Vieira, M. A. M., and Loureiro, A. A. (2017a). Mobile Matrix: A Multihop Address Allocation and Any-to-Any Routing in Mobile 6LoWPAN. In Proceedings of the 13th ACM Symposium on QoS and Security for Wireless and Mobile Networks, Q2SWinet'17, pages 65-72.

Santos, B. P., Rettore, P. H., Ramos, H. S., Vieira, L. F., and Loureiro, A. A. (2017b). T-MAPS: Modelo de Descrição do Cenário de Trânsito Baseado no Twitter. Anais do XXXV Simpósio Brasileiro de Redes de Computadores e Sistemas Distribuídos.

Santos, B. P., Rettore, P. H., Ramos, H. S., Vieira, L. F., and Loureiro, A. A. (2018b). Enriching Traffic Information with a SpatiotemporalModel based on Social Media. In Computers and Communications (ISCC), 2018 IEEE Symposium on, page 0. IEEE.

Santos, B. P., Rettore, P. H. L., Vieira, L. F. M., and Loureiro, A. A. (2018c). Dribble: a learn-based timer scheme selector for mobility management in iot. IEEE Wireless Communications and Networking Conference WCNC.

Santos, B. P., Silva, L. A., Celes, C., Borges, J. B., Neto, B. S. P., Vieira, M. A. M., Vieira, L. F. M., Goussevskaia, O. N., and Loureiro, A. (2016). Internet das coisas: da teoria à prática. Minicursos SBRC-Simpósio Brasileiro de Redes de Computadores e Sistemas Distribuıdos, 31 .

Santos, B. P., Vieira, L. F. M., and Vieira, M. A. M. (2017c). CGR: Centrality-based green routing for Low-power and Lossy Networks. Computer Networks, 129:117-128.

Santos, B. P., Vieira, M. A. M., and Vieira, L. F. M. (2015). eXtend collection tree protocol. In Wireless Communications and Networking Conference (WCNC), 2015 IEEE, pages 1512-1517. IEEE.

Vasseur, J.-P. and Dunkels, A. (2010). Interconnecting smart objects with ip: The next internet. Morgan Kaufmann.

Winter, T., Thubert, P., Brandt, A., Hui, J., Kelsey, R., Levis, P., Pister, K., Struik, R., Vasseur, J., and Alexander, R. (2012). RPL: IPv6 Routing Protocol for Low-Power and Lossy Networks. RFC 6550 (Proposed Standard). 